Issued by Sandia National Laboratories, operated for the United States Department of Energy by Sandia Corporation.

NOTICE: This report was prepared as an account of work sponsored by an agency of the United States Government. Neither the United States Government, nor any agency thereof, nor any of their employees, nor any of their contractors, subcontractors, or their employees, make any warranty, express or implied, or assume any legal liability or responsibility for the accuracy, completeness, or usefulness of any information, apparatus, product, or process disclosed, or represent that its use would not infringe privately owned rights. Reference herein to any specific commercial product, process, or service by trade name, trademark, manufacturer, or otherwise, does not necessarily constitute or imply its endorsement, recommendation, or favoring by the United States Government, any agency thereof, or any of their contractors or subcontractors. The views and opinions expressed herein do not necessarily state or reflect those of the United States Government, any agency thereof, or any of their contractors.

Printed in the United States of America. This report has been reproduced directly from the best available copy.

Available to DOE and DOE contractors from Office of Scientific and Technical Information P.O. Box 62

Oak Ridge, TN 37831

Prices available from (703) 605-6000

Web site: http://www.ntis.gov/ordering.htm

Available to the public from National Technical Information Service U.S. Department of Commerce

5285 Port Royal Rd

Springfield, VA 22161

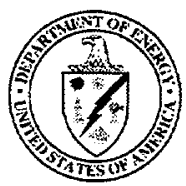




\section{DISCLAIMER}

Portions of this document may be illegible in electronic image products. Images are produced from the best available original document. 
SAND2000-0240

Unlimited Release

Printed February 2000

\title{
Final Report for the Designed Synthesis of Controlled Degradative Materials LDRD
}

\author{
D. A. Loy, T. A. Ulibarri, J. Curro, R. Saunders, D. Derzon, T. Guess, B. M. Baugher \\ Catalysts and Chemical Technologies Department \\ Sandia National Laboratories \\ P.O. Box 5800 \\ Albuquerque, NM 87185-1407
}

\begin{abstract}
The main goal of this research was to develop degradable systems either by developing weaklink-containing polymers or identifying commercial polymeric systems which are easily degraded. In both cases, the degradation method involves environmentally friendly chemistries. The weaklinks are easily degradable fragments which are introduced either randomly or regularly in the polymer backbone or as crosslinking sites to make high molecular weight systems via branching. We targeted three general application areas: 1) non-lethal deterrents, 2) removable encapsulants, and 3) readily recyclable/environmentally friendly polymers for structural and thin film applications.
\end{abstract}





\section{Contents}

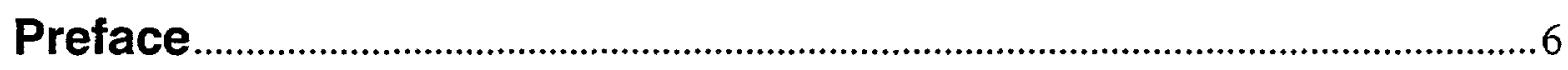

Section 1. Introduction to Designed Synthesis of Controlled Degradative Materials LDRD

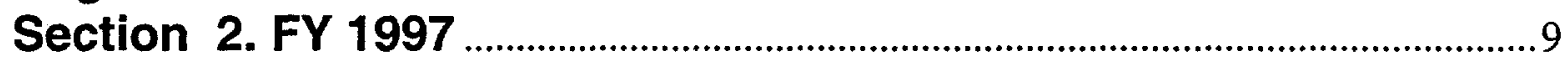

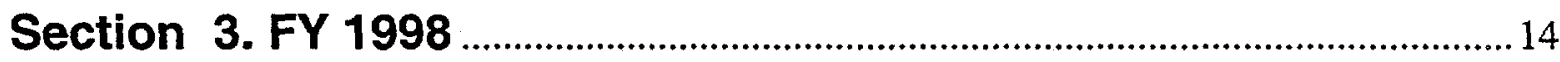

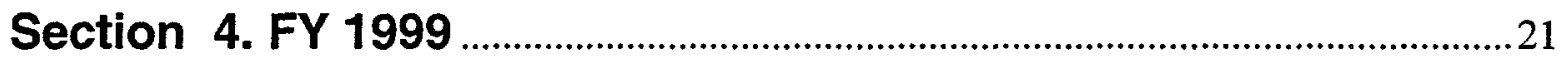

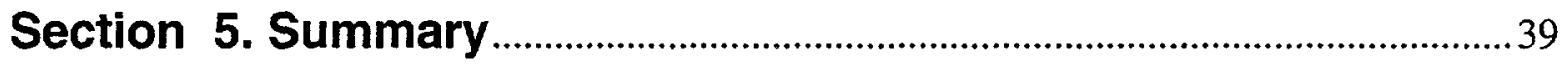




\section{Preface}

The final report is broken down into four sections, an introduction and one for each year of the project. Within each section, there are Materials Development, Materials Testing, and Molecular Modeling subsections:

- Section 1 - Introduction

- Section 2 - Results for FY 97

- Section 3 - Results for FY 98

- Section 4 - Results for FY 99

- Section 5 - Summary 
SECTION 1

Introduction to Designed Synthesis of Controlled Degradative Materials LDRD 
The main goal of this research was to develop degradable systems either by developing weaklink-containing polymers or identifying commercial polymeric systems which are easily degraded. In both cases, the degradation method involves environmentally friendly chemistries. The weak-links are easily degradable fragments which are introduced either randomly or regularly in the polymer backbone or as crosslinking sites to make high molecular weight systems via branching. We targeted three general application areas: 1) non-lethal deterrents, 2) removable encapsulants, and 3) readily recyclable/environmentally friendly polymers for structural and thin film applications. 


\section{SECTION 2}

\section{Results for FY 97}

\section{Materials Development}

The successful synthesis of a variety of low-moderate molecular weight weaklink containing polymers has been achieved. These materials contain the weaklink moiety both in a random fashion and at a regular spacing. To date, the nature of the weaklink 
placement (random or regular) has not been shown to be critical. The most important factor is the general spacing between the weak links, such that the degraded material is of sufficiently low molecular weight to ensure high solubility and therefore removability. While the synthesis of photo- and thermally degradable polymers is still being pursued in this project, the best results to date have been in the synthesis of polymers which are susceptible to chemical or enzymatic (esterase) degradation. Successful degradation has already been demonstrated for a chemically degradable (dilute base) system (9/97 milestone). The degradation characteristics of other functionalized systems will be investigated during the remainder of this fiscal year.

The synthetic work this year has shown that one risk to this project that we had not predicted was the ability to achieve the extremely high molecular weights that are needed to obtain optimal properties for our first application (in this case an extremely low coefficient of friction or high lubricity). While recent results have demonstrated that this difficulty is surmountable and high molecular weight materials can be generated, it highlights potential difficulties for commercialization and application in the timely manner that potential customers would desire for first generation materials. Therefore, we propose to add an additional thrust to our synthetic effort, an approach utilizing commercially available polymer systems which are treated to promote degradation vulnerability.

While the general goal of the polymer industry is to manufacture extremely robust systems, many commercial polymers are known to be incompatible with certain environments. For example, polyacetals, which are also known to generate surfaces with low coefficients of friction, are susceptible to both acid and base degradation. In 
fact, strong oxidizing agents, such as hypochlorite (bleach) at concentrations greater than $1 \mathrm{ppm}$ in water can deteriorate some polyacetals. To accelerate photosensitive systems, photo- and singlet-oxygen sensitizers can be blended with polymers to make degradable systems. The overall aim of this portion of the work will be to determine the effectiveness of blended formulations based on susceptible commercial polymers for producing a first generation material for applications in a more timely fashion.

\section{Materials Testing}

In order to screen the weak link materials for the super-slick application, a torsion shear test method was developed to measure and compare the lubricity of the various water-soluble polymer systems being synthesized in this program. This test method is the culmination of several iterations with different sample preparation techniques and platen geometries. The shear test technique consists of measuring the torque required to rotate a cylindrical metal platen that has one end in contact with a polymer powder/water mixture. The torque level is a quantitative indicator of the lubricity (coefficient of friction) of the polymer system. The torque vs. time curves which are generated (Figure 1) are utilized to distinguish differences in the lubricity of different super-slick polymers. The method can be used to differentiate the effects of different variables (e.g., polymer chemistry, molecular weight, platen material, platen surface roughness, etc.) on the measured lubricity.

\section{Molecular Modeling:}


In order to predict the structure and the properties (i.e., surface tension) of our polymer systems at a surface, wall PRISM (Polymer Reference Interaction Site Model) calculations were completed. In addition, a more rigorous computational technique involving density functional theory (DFT) was developed and used to predict the interfacial structure. This DFT methodology was successfully demonstrated for a liquid of freely-joined polyethylene oxide chains near a wall. It was found that there is a distinct change in the structure or density profile of the polymer at the interface, as well as a change in the interface thickness, with changing molecular weight (Figure 2). These structural changes correlate with the change in surface properties measured in our torsion shear test method. 


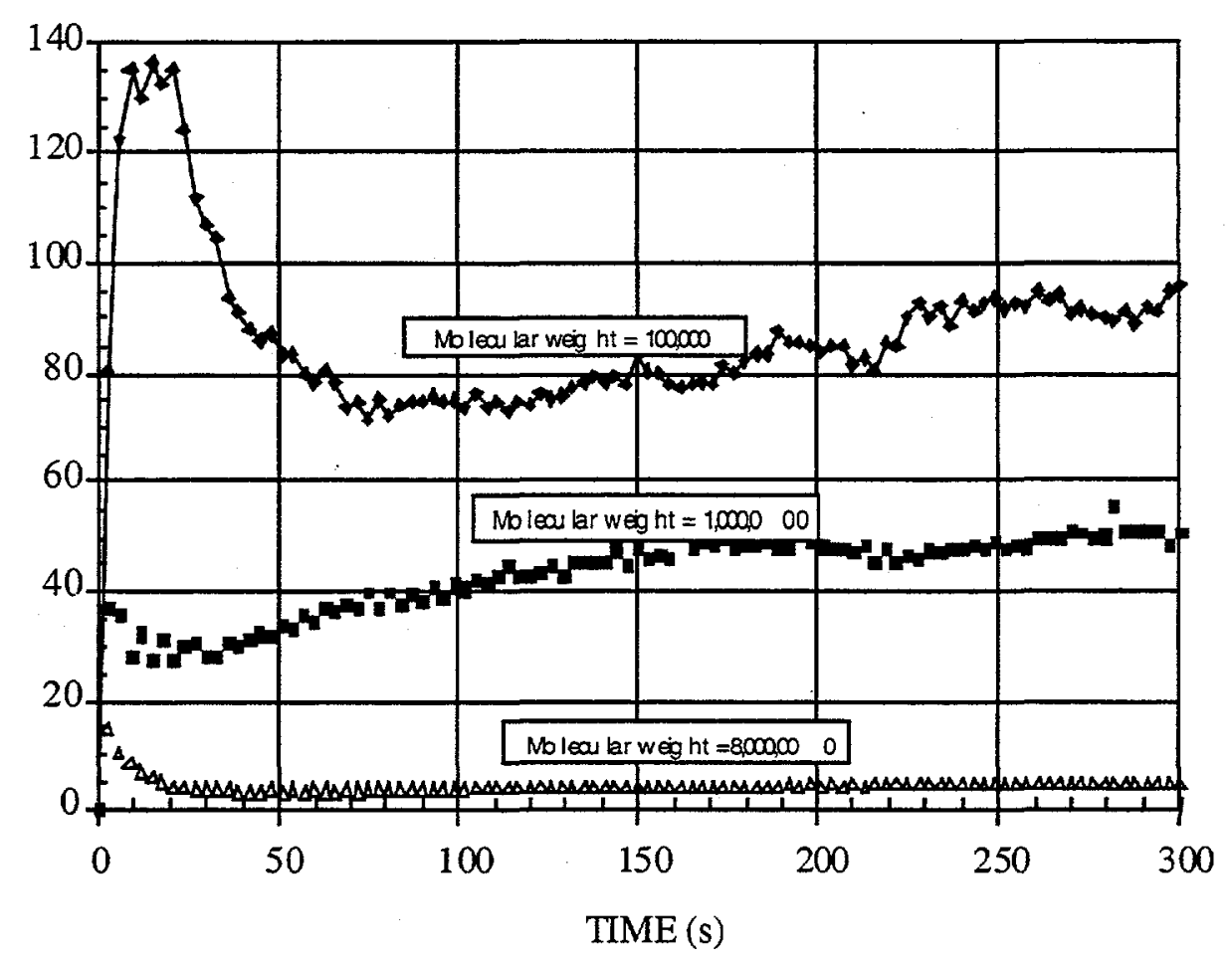

Figure 1. Torque vs. time curves for polyethylene oxide at three different molecular weights.

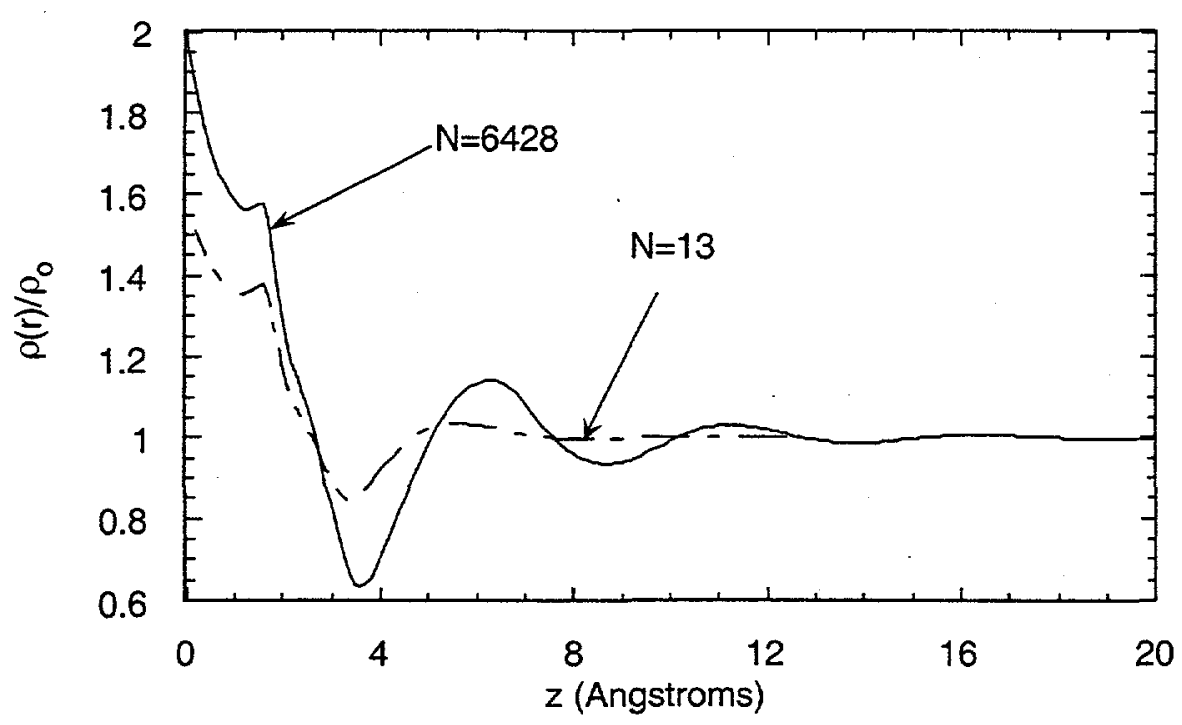

Figure 2. Plot of the density profile of a polyethylene polymer versus distance from a surface for different molecular weight polymers. 
SECTION 3

Results for FY 98 


\section{Materials Development and Mechanical Testing}

A variety of new linear and branched synthesis methods have been utilized to generate the needed high molecular weight polymers (1/98 milestone). The most successful method for forming weak link containing high molecular weight systems suitable for degradation involves the formation of branched systems. This approach involves attaching pendant groups along the polymer backbone which are amenable to crosslinking reactions with short polymer fragments. The two major benefits of this method above and beyond its effectiveness in providing high molecular weight products are, 1) it allows the introduction of a wide variety of short chain crosslink fragments leading to a breadth of possible polymer product applications, and 2) it allows facile entry into both chemical and thermal weak link systems. Our most successful system for super-slick applications involves a base degradable branched copolymer containing polyacrylamide and polyethylene oxide (PEO) segments (3/98 milestone). The polyacrylamide system was chosen for its water solubility as well as its potential propensity to aid in obtaining a low coefficient of friction. This water soluble system has been characterized and mechanically tested using the torsion shear test method developed in FY97 (Figure 3) (5/98 milestone). The shear test technique consists of measuring the torque required to continuously rotate a cylindrical metal platen that has one end in contact with the polymer/water mixture. Sample preparation consisted of introducing the polymer to water and crushing the polymer to form a uniform mixture. The torque level is a quantitative indicator of the lubricity (coefficient of friction) of the polymer/water mixture. The polymer A1 is the pure polyacrylamide, A2 contains 2.5 wt\% PEO, A3 contains 4.8 wt $\%$ PEO, and A4 contains 7.0 wt\% PEO. 


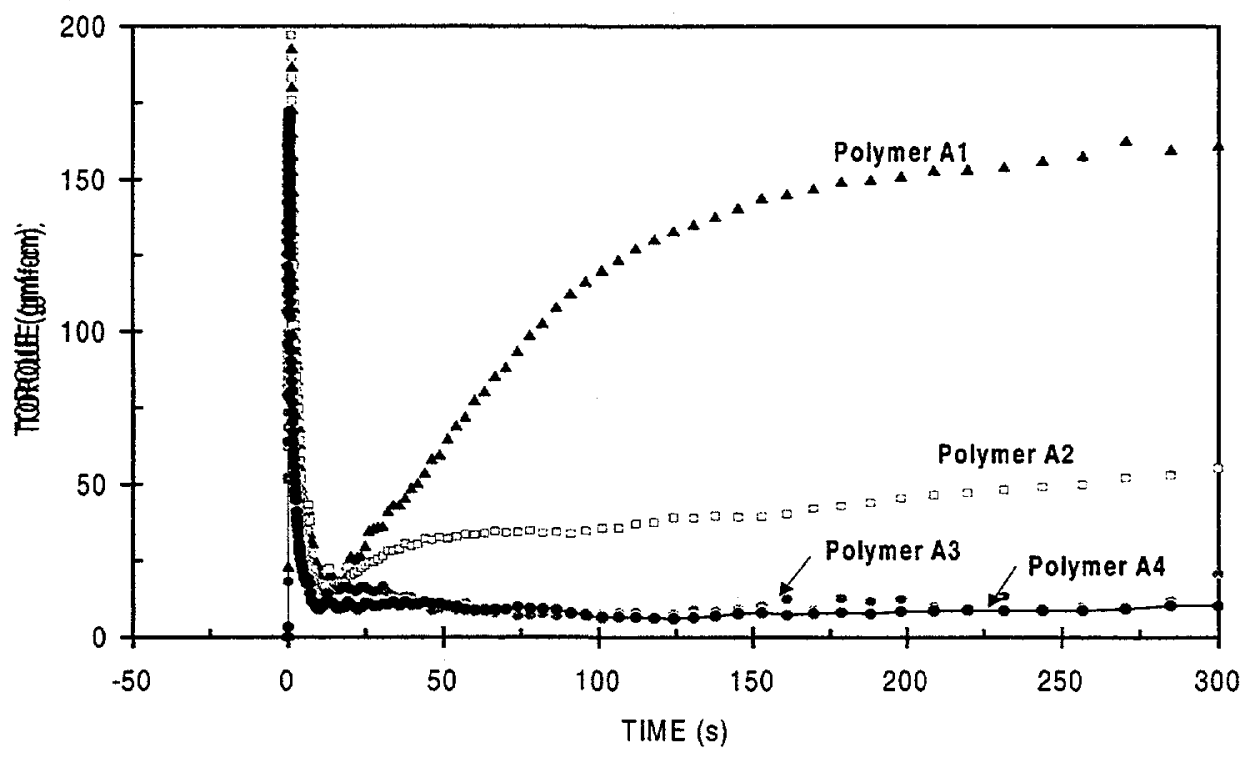

Figure 3. Plot of torque (gmf-cm) vs. rotation time (s) for polyacrylamide/PEO weak link systems.

The torque vs. time curves in Figure 1 compare the lubricity of the four weak link super-slick polymers. (Note that there is an initial high torque as the cylinder begins spinning due to overcoming static friction.) Comparing the Al-A4 polymers to PEO, which is the standard super-slick polymer of choice (Table 1), indicates that A2 and 1 million MW PEO have essentially the same lubricity. A3 and A4 have approximately the same lubricity value and are approaching 8 million MW PEO. Ongoing work is currently evaluating the degradation characteristics of A3 and A4 (9/98 milestone). 
Table 1. Lubricity values for PEO and copolymer systems.

Polymer Torque @ 300s (ave.) Polymer Torque @ 300s (ave.)

$\begin{array}{llll}\text { A1 } & 158 & \text { PEO (1K MW) } & 96 \\ \text { A2 } & 55 & \text { PEO (1 million MW }) & 51 \\ \text { A3 } & 17 & \text { PEO (8 million MW }) & 5 \\ \text { A4 } & 10 & & \end{array}$

In order to produce degradable super-slick systems for more immediate use in deterrent applications, commercial polymers have been examined. Initial systems under consideration were polyacetals, polyalcohol/metal oxide systems and PEO itself (4/98 milestone). Considering the highly effective nature of PEO for providing a super-slick surface and promising literature information, this part of the study concentrated on degrading PEO. In order to quickly screen weak link materials for super-slick application and degradation, a laboratory test was developed (the torsion test system is not amenable to acid and base introduction). The system is comprised of a MINIMAT Miniature Materials Tester manufactured by Polymer Laboratories and custom fixturing. The fixturing is set up to measure the amount of shear force needed to pull a weighted ( 2 lb), sand-blasted aluminum block over a surface of Buehler 320 grit sandpaper. The force required to pull the weighted block is related to the lubricity of the tested 
polymeric materials. Baseline measurements with dry and water wetted sandpaper were done. Testing involved introducing the polymer in a sieve or salt shaker fashion. A uniform layer of powdered polymer was deposited onto the sandpaper and then misted with water. After the desired gel time was reached, the block was pulled across the gel surface a length of $10 \mathrm{~mm}$. The most effective and environmentally friendly method of degrading PEO was found to be using a dilute ascorbic acid (vitamin C) solution (Figure $4)$.

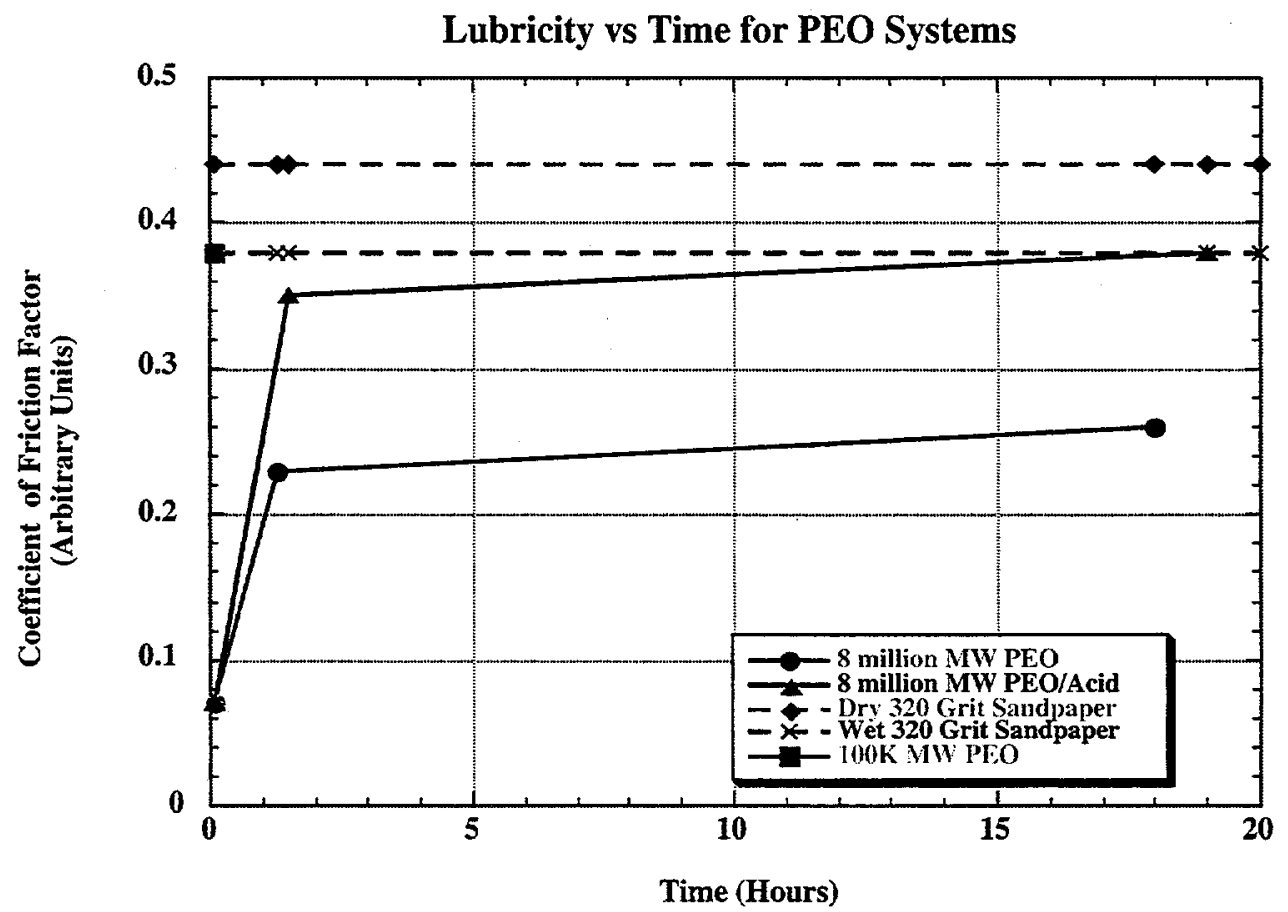

Figure 4. Plot of coefficient of friction factor (MINIMAT test) vs. polymer wetting time.

The bench top friction measurements indicate that 100K MW PEO has the same lubricity as water; defining a limiting molecular weight which must be exceeded to 
obtain nominal super-slick characteristics. For 8 million MW PEO, there is a definite initial change in lubricity with gel time, however, the polymer maintains a low coefficient of friction. Treatment of the 8 million MW PEO with dilute ascorbic acid leads to a marked decrease in lubricity after 1.5 hours and a complete loss in super-slick properties in 19 hours. Therefore, the PEO/ascorbic acid pair provides a removable super-slick deterrent system which, while not a lock and key system, is potentially deployable for more immediate use. Final characterization with respect to deployment processes are ongoing (8/98 milestone).

Molecular Modeling: For super-slick applications, it is important that the polymer have a high degree of lubricity. Although the detailed mechanism of how a water soluble polymer like PEO increases lubricity is not known, recent studies suggest that a high degree of lubricity can be achieved when a liquid film de-wets a surface. Under dewetting conditions a liquid film or drop undergoes a "tractoring" phenomenon akin to a drop rolling down an incline rather than spreading across a surface. Whether the polymer solution wets a surface or not is determined by the surface tension of the liquid. For this reason, we performed a preliminary series of Density Functional Theory (DFT) calculations on a polymer chain liquid near a surface to identify the factors that are important in controlling the surface tension. Polymer density, $\rho$ chain stiffness (persistence length), and the intermolecular attractions $\varepsilon / \mathrm{kT}$ (cohesive forces) were varied in a DFT calculation on a semiflexible chain model equivalent to a degree of polymerization of approximately 50 monomer units. As can be seen in Figure 5 , the surface tension appears insensitive to both polymer density and backbone stiffness. The 
cohesive forces, on the other hand, play a critical role in determining the surface tension. For strongly attracting polymer chains, the macromolecules tend to maximize the intermolecular contacts thus increasing the surface tension or free energy to create a surface (5/98 milestone).

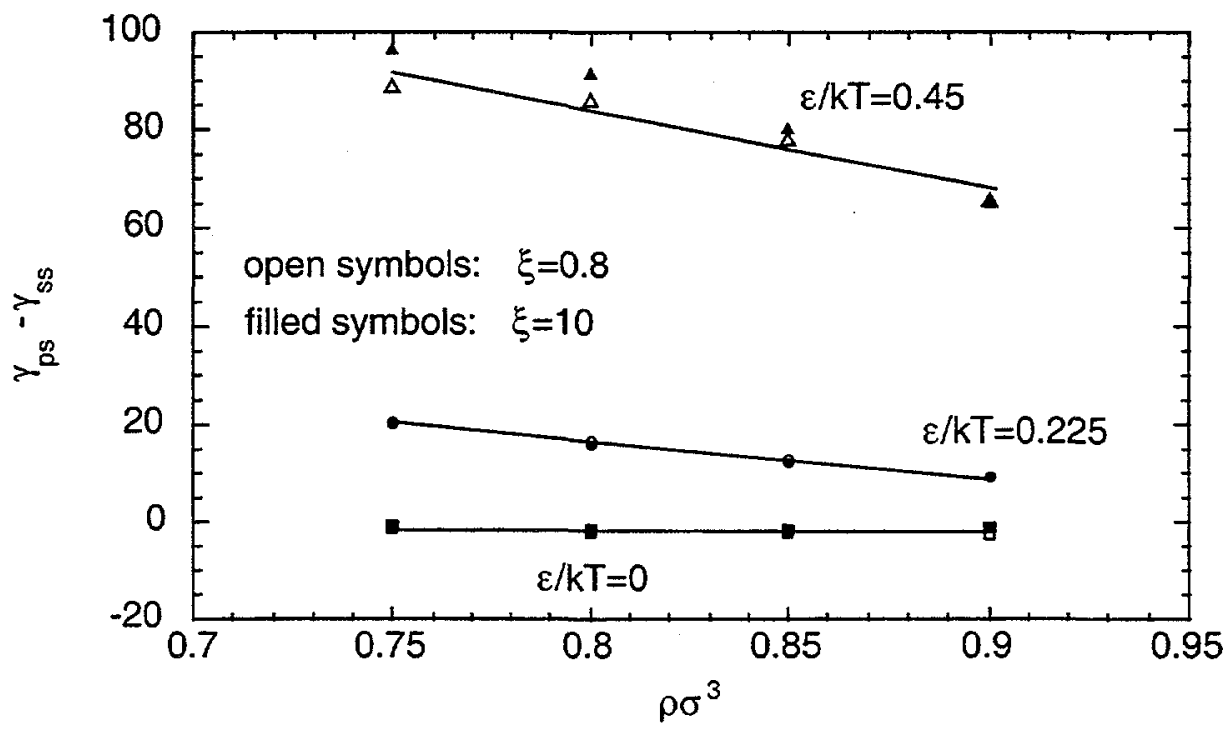

Figure 5. Calculations looking at surface tension dependence on polymer density, chain stiffness and cohesive energy. 


\section{SECTION 4}

\section{Results for FY 99}

Materials Development 
A new class of removable encapsulants based on organosilane precursors functionalized with maleimide groups were developed. These materials would thermally cure at room temperature without catalysts or reagents and would depolymerize to low molecular constitutents at temperatures at or above ninety degrees Celsius.
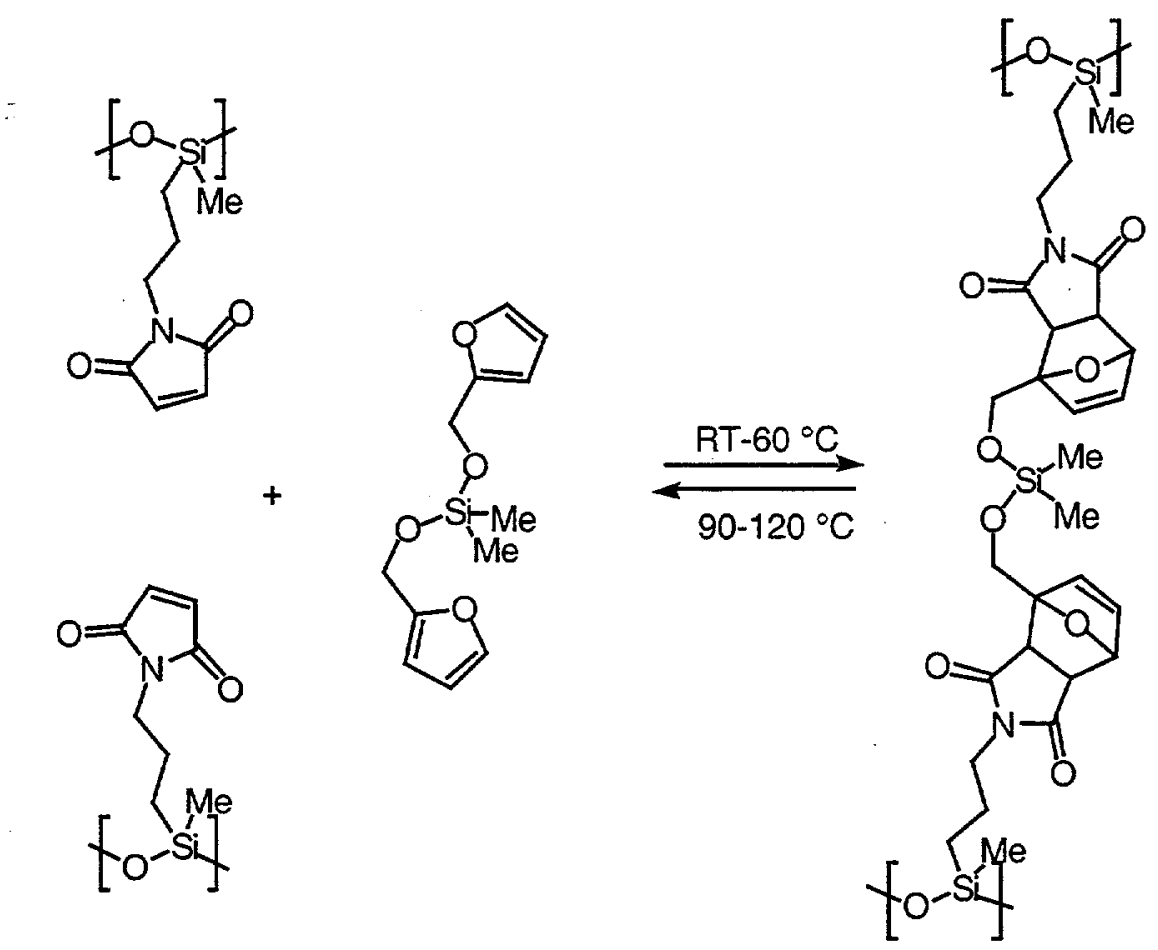

Figure 6. Polymers with thermally labile weaklink.

These new materials could be irreversibly cured by auto-polymerization with or without a radical generating catalyst or through Alder-ene chemistry. 


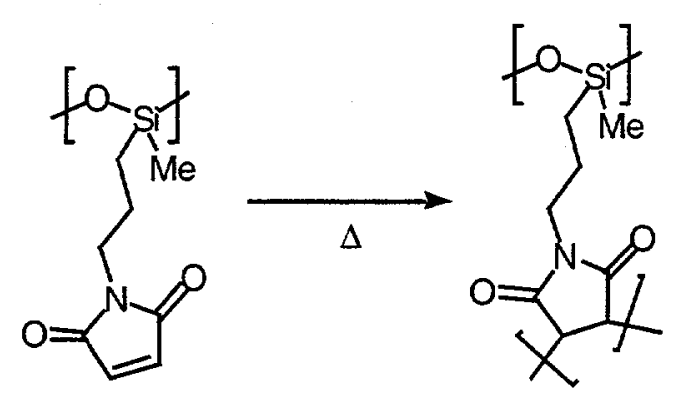

Figure 7. Irreversible free-radical or thermal crosslinking through the maleimde functionality.

The monomers are also very promising as a new class of coupling agents for coupling unsaturated polymers such as rubbers to silica fillers.

\section{Materials Testing}

A survey of the literature revealed that ascorbic acid would quickly degrade the slipperiness of polyethylene oxide. We proposed that the ascorbic acid was generating radicals that would cause cleavage of the PEO into shorter thengths. It has been shown earlier in this LDRD that the slipperiness of the hydrated PEO is directly dependent on molecular weight with a critical threshold of around 1 million Daltons being necessary for a slippery surface. We proposed that this radical-induced cleavage could rapidly degrade high molecular weight PEO to lower molecular weight material with a parallel decrease in performance. There is considerable evidence that ascorbic acid degrades PEO through the generation of hydroxide radicals that then abstract an alpha hydrogen from the polymer. This leads to chain scission of the PEO lowering the mean molecular weight. We were able to confirm this hypothesis with viscosity studies on aqueous 
solutions of high moelcular weight (8 Million Daltons) PEO with $25 \mathrm{wt} \%$ ascorbic acid. Within 1000 seconds the molecular weight had decreased to 1 million Daltons. After two hours the weight was down to 250,000 Daltons. Interestingly, hydrogen peroxide or photo-radical generators like benzophenone-triethyl amine did not result in any measurable degradation of the PEO. Nor were most organic (organic soluble) radical generators sufficiently soluble in the aqueous solutions for any measurable degradation to be observed.

In order to attempt to prevent degradation of slipperiness with ascorbic acid, alternative polymers based on lightly crossslinked polyacryalmides were synthesized and evaluated for slipperiness. The polyacrylamde crosslinked with ethylene diacrylate $(2-3 \mathrm{~mol} \%)$ were shown to have slipperiness comparable with high molecular weight PEO. Those based on bis(acrylamide) crosslinkers were not slippery. Degradation tests were not performed.

\section{Molecular Modeling}

\section{Introduction}

The addition of small amounts of soluble polymers, such as polyethylene oxide, to water has the effect of making the solution "slippery". Why this happens is puzzling. The addition of polymer would be expected to increase the viscosity of the solution resulting in reduced lubricity. Furthermore, one would expect that the addition of the polymer would lower the surface tension and increase the wettability of the solution against a substrate. Again, increased wetting of the surface might be expected to increase, not decrease the friction forces with the surface. In the case of soap solutions, 
the soap molecules act as a surfactant and form a soap layer at which the substrate can slide. Polyethylene oxide, however, does not have a surfactant-like character with a polar head group and hydrophobic tail. Therefore, the mechanism by which these polymers reduce friction at a surface is not well understood. The purpose of this modeling study is aimed at this question.

Presumably, the superslick mechanism must somehow be related to the structure of the interface between the polymer solution and the substrate. Therefore, the current modeling study has been directed towards a more complete understanding of the equilibrium structure and thermodynamics of inhomogeneous systems with the intent of investigating wetting and adhesion issues; however, numerous other applications in both traditional and non-traditional polymers can be imagined.

Density functional (DFT) theory was the methodology adopted for this study, and before describing our results, we will briefly review the basic theory. The full results of our study were reported in three articles ${ }^{1-3}$, two of which are currently under review with the Journal of Chemical Physics and the third will also be submitted in the near future.

\section{Density Functional Theory}

The excess Helmholtz free energy of the inhomogeneous system, $A-A_{0}$ where the ' 0 ' indicates the ideal system, is expanded through second order about the bulk, homogeneous system as 


$$
\begin{aligned}
& \left(\mathrm{A}-\mathrm{A}_{0}\right)=\left(\mathrm{A}-\mathrm{A}_{0}\right)_{\text {bulk }}+\sum_{\mathrm{i}} \int_{\mathrm{V}} \mathrm{d} \underline{\mathrm{r}}\left[\Psi_{\mathrm{i}}(\underline{\mathrm{r}})-\psi_{\mathrm{i}}^{0}(\underline{\mathrm{r}})\right]_{\text {bulk }} \Delta \rho_{\mathrm{i}}(\underline{\mathrm{r}}) \\
& +\left.\frac{1}{2} \sum_{i, j} \iint_{V} d \underline{r} d \underline{r}^{\prime} \frac{\delta^{2}\left(\mathrm{~A}-\mathrm{A}_{0}\right)}{\delta \rho_{\mathrm{i}}(\underline{\mathrm{r}}) \delta \rho_{\mathrm{j}}\left(\underline{\underline{r}}^{\prime}\right)}\right|_{\text {bulk }} \Delta \rho_{\mathrm{i}}(\underline{\mathrm{r}}) \Delta \rho_{\mathrm{j}}\left(\underline{\underline{r}}^{\prime}\right)
\end{aligned}
$$

where the conjugate variable to the density, $\rho_{\mathrm{i}}(\mathrm{r})$, is the "external field" or "spatially varying chemical potential", $\psi_{\mathrm{i}}(\mathrm{r})=\mu_{\mathrm{i}}-\mathrm{U}_{\mathrm{i}}(\mathrm{r}) ; \mu_{\mathrm{i}}$ is the usual chemical potential; and $\mathrm{U}_{\mathrm{i}}(\mathrm{r})$, the external field. The sums are over site type, and the integrals, over the system volume. The coefficients in the functional expansion are evaluated in the bulk state; and the expansion variables, $\Delta \rho_{i}(r)$, equal $\rho_{\mathrm{l}}(\mathrm{r})-\rho_{\mathrm{l}, \text { bulk. }}$. The thermodynamic identity

$$
\psi_{\mathrm{i}}(\underline{\mathrm{r}})=\frac{\delta \mathrm{A}}{\delta \rho_{\mathrm{i}}(\underline{\mathrm{r}})}
$$

was used to simplify the first order term.

In applications, the fields, $\psi_{\mathrm{i}}(\mathrm{r})$, rather than the densities are the independent variables. As a result, it is more convenient to work in terms of the grand potential functional, $\mathrm{W}$, which is found from the Legendre transform

$$
\mathrm{W}=\mathrm{A}-\sum_{\mathrm{i}} \int_{\mathrm{V}} \mathrm{d} \underline{\underline{r}} \Psi_{\mathrm{i}}(\underline{\mathrm{r}}) \mathrm{p}(\underline{\underline{r}})
$$

This, combined with equation (2.1) results in

$$
\begin{aligned}
\Delta \mathrm{W}= & \Delta \mathrm{W}_{0}-\sum_{\mathrm{i}} \int_{\mathrm{V}} \mathrm{d} \underline{\mathrm{r}}\left[\psi_{\mathrm{i}}(\underline{\mathrm{r}})-\psi_{\mathrm{i}, \text { bulk }}\right]_{\mathrm{i}}(\underline{\mathrm{r}}) \\
& +\sum_{\mathrm{i}} \int_{\mathrm{V}} d \underline{\underline{r}}\left[\Psi_{\mathrm{i}}^{0}(\underline{\mathrm{r}})-\psi_{\mathrm{i}, \text { bulk }}^{0}\right]_{\mathrm{i}}(\underline{\mathrm{r}}) \\
& +\left.\frac{1}{2} \sum_{\mathrm{i}, \mathrm{j} V \mathrm{~V}} \int \underline{\mathrm{d}}_{\mathrm{r}} \mathrm{d} \underline{\underline{r}}^{\prime} \frac{\delta^{2}\left(\mathrm{~A}-\mathrm{A}_{0}\right)}{\delta \rho_{\mathrm{i}}(\underline{\mathrm{r}}) \delta \rho_{\mathrm{j}}\left(\underline{\mathrm{r}}^{\prime}\right)}\right|_{\text {bulk }} \Delta \rho_{\mathrm{i}}(\underline{\mathrm{r}}) \Delta \rho_{\mathrm{j}}\left(\underline{\mathrm{r}}^{\prime}\right)
\end{aligned}
$$


where the $\Delta \mathrm{W}=\mathrm{W}-\mathrm{W}_{\text {bulk }}$.

At this point, the expression for $\Delta \mathrm{W}$ is completely general since the ideal system has not been defined. Indeed, if no ideal system is used, the minimum of equation (2.4) is found in Fourier space to be

$$
\hat{\mathrm{g}}(\underline{\mathrm{k}})=\delta(\mathrm{k}) \underline{\mathrm{g}}_{\mathrm{bulk}}-\left[\underline{\hat{\mathrm{A}}}_{\mathrm{bulk}}^{\prime}(\underline{\mathrm{k}})\right]^{-1} \cdot \hat{\mathrm{U}}(\underline{\mathrm{k}})
$$

where matrix notation is used; $\mu$ is set equal to $\mu_{\text {bulk }}$; and A" is the second derivative of the Helmholtz free energy indicated in equation (2.4) with $A_{0}=0$. Clearly, this is a poor approximation except for very small fields.

If an ideal monatomic gas is taken as the ideal system, the densities and fields are related by

$$
\begin{aligned}
\rho_{i}(\underline{r}) & =\rho_{i, \text { bulk }} \exp \left[-\beta U_{i}^{0}(\underline{r})_{-}^{-}\right. \\
& =\exp \left[\beta \psi_{i}^{0}(\underline{r})\right]
\end{aligned}
$$

where $\beta$ is $1 / \mathrm{kT} ; \mathrm{k}$ is the Boltzmann constant; $\mathrm{T}$ is the temperature; and the bulk chemical potential is set to $k \operatorname{Tln}\left(\rho_{\mathrm{i}, \text { bulk }}\right)$. Through equation (2.2), the Helmholtz free energy difference can be found to be

$$
\Delta \beta A_{0}=\sum_{i} \int_{V} d \underline{r} \rho_{i}(r)\left[\ln \left(\rho_{i}(r)\right)-1\right]-\sum_{i} \int_{V} d r \mid \rho_{i, b u l k}\left[\ln \left(\rho_{i, b u l k}\right)-1\right]
$$

or, as is expected from thermodynamics,

$$
\Delta \beta \mathrm{W}_{0}=-\sum_{\mathrm{i}} \int_{\mathrm{V}} \mathrm{d} \underline{\mathrm{r}} \Delta \rho_{\mathrm{i}}(\underline{r})
$$

Equations (2.6) and (2.8) in combination with equation (2.1) result in a minimum of 
$\rho_{i}(\underline{r})=\rho_{i, \text { bulk }} \exp \left[-\beta U_{i}(\underline{r})-\sum_{j} \int_{V} d \underline{r}^{\prime}\left(A-A_{0}\right)_{i, j, b u l k}^{\prime \prime}\left(\underline{r}-\underline{r}^{\prime}\right) \Delta \rho_{j}\left(\underline{r}^{\prime}\right)\right]$

which is a vast improvement on equation (2.5), but only includes bonding constraints weakly through $\left(\mathrm{A}-\mathrm{A}_{0}\right)$ "'.

The ideal system we use is at the next level of complexity where bonding is explicitly included in the ideal system:

$\rho_{\mathrm{i}}(\underline{r})=\rho_{\mathrm{i}, \text { bulk }} \sum_{\mathrm{k}}^{\prime} \int \cdots \int \mathrm{d} \underline{\underline{r}}_{1} \cdots \mathrm{d} \underline{\mathbf{r}}_{\mathrm{k}-1} \mathrm{~d} \underline{\underline{r}}_{\mathrm{k}+1} \cdots \mathrm{d} \underline{\mathbf{r}}_{\mathrm{N}} \exp \left[-\beta \sum_{\mathrm{j}}{ }^{\prime} \mathrm{U}_{\mathrm{j}}^{0}\left(\underline{\underline{r}}_{\mathrm{j}}\right)\right] \mathrm{S}\left(\underline{\mathbf{r}}_{1}, \cdots, \underline{\mathbf{r}}_{\mathrm{N}}\right)$

where $n_{i}$ is the number of sites of type " $i$ "; the sum $\Sigma$ ' is over all sites of type " $i$ " on a single chain; the sum $\Sigma^{\prime \prime}$ is over all sites on a single chain; and $S\left(\mathrm{r}_{1}, \ldots, \mathrm{r}_{\mathrm{N}}\right)$ denotes the bonding constraints upon the chain. In terms of the $\psi^{0 \text { s, }}$, this becomes

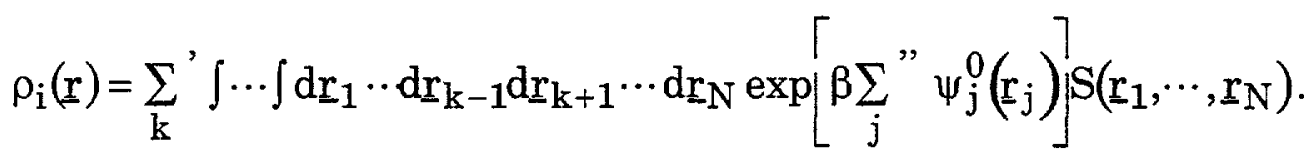

By using the thermodynamic relationship

$$
-\rho_{i}(\underline{r})=\frac{\delta W}{\delta \psi_{i}(\underline{r})},
$$

the expression for $\Delta \mathrm{W}^{0}$ is found to be

$$
\Delta \beta \mathrm{W}_{0}=-\frac{1}{\mathrm{~N}} \sum_{\mathrm{i}} \int_{\mathrm{V}} \mathrm{d} \underline{\mathrm{r}} \Delta \rho_{\mathrm{i}}(\underline{\mathrm{r}})
$$

where $\mathrm{N}$ is the total number of sites on a chain.

Now, equation (2.2) in combination with equation (2.13) must be minimized with respect to $\rho_{\mathrm{i}}(\mathrm{r})$; however, since the $\psi^{0}$ s cannot be completely removed from the expression for $\Delta \mathrm{W}$, a method related to that of undetermined multipliers is used. 
Usually, a $\lambda(r)$ function would be introduced to ensure that the minimum of $\Delta W$ with respect to both $\rho(\mathrm{r})$ and $\psi^{0}(\mathrm{r})$ would coincide to the constraint of equation (2.11). In the present case this insurance can be achieved in a slightly different manner by substituting equations (2.11) and (2.13) into the $\Delta \mathrm{W}$ expression to get

$$
\begin{aligned}
& \Delta \mathrm{W}=-\mathrm{kT} \int \cdots \int \mathrm{d} \underline{\underline{r}}_{1} \cdots \mathrm{d} \underline{\underline{r}}_{\mathrm{N}} \exp \left[\beta \sum_{\mathrm{j}} ” \psi_{j}^{0}\left(\underline{r}_{j}\right)\right] \mathrm{S}\left(\underline{r}_{1}, \cdots, \underline{r}_{N}\right)+\frac{\mathrm{kTV}}{\mathrm{N}} \sum_{\mathrm{i}} \rho_{\mathrm{i}, \text { bulk }}- \\
& -\sum_{i} \int_{V} d \underline{r}\left[\psi_{i}(r)-\psi_{i, b u l k} b_{i}(\underline{r})+\sum_{i} \int_{V} d \underline{r}\left[\psi_{i}^{0}(\underline{r})-\psi_{i, b u l k}^{0}\right] b_{i}(\underline{r})\right. \\
& +\left.\frac{1}{2} \sum_{i, j \mathrm{~V}} \iint_{\mathrm{V}} \mathrm{d} \underline{\mathbf{r}}^{\mathrm{d}} \underline{\underline{r}}^{\prime} \frac{\delta^{2}\left(\mathrm{~A}-\mathrm{A}_{0}\right)}{\delta \rho_{\mathrm{i}}(\underline{\mathbf{r}}) \delta \rho_{\mathrm{j}}\left(\underline{\mathbf{r}}^{\prime}\right)}\right|_{\text {bulk }} \Delta \rho_{\mathrm{i}}(\underline{\mathbf{r}}) \Delta \rho_{j}\left(\underline{\mathbf{r}}^{\prime}\right)
\end{aligned}
$$

Here the partial functional derivative with respect to $\psi^{0}(\mathrm{r})$ enforces the constraint equation without an explicit $\lambda(r)$.

The derivative of $\Delta W$ with respect to $\rho(r)$ yields the expression for the ideal field

$$
\left[\psi_{i}^{0}(\underline{r})-\psi_{i, \text { bulk }}^{0}\right]=\left[\psi_{i}(\underline{r})-\psi_{i, \text { bulk }}\right]-\left.\sum_{j} \int_{V} d \underline{r}^{\prime} \frac{\delta^{2}\left(\mathrm{~A}-\mathrm{A}_{0}\right)}{\delta \rho_{\mathrm{i}}(\underline{\mathrm{r}}) \delta \rho_{\mathrm{j}}\left(\underline{r}^{\prime}\right)}\right|_{\text {bulk }} \Delta \rho_{j}\left(\underline{r}^{\prime}\right) .
$$

If the chemical potentials are set equal in inhomogeneous and homogeneous systems, this simplifies to

$$
U_{i}^{0}(\underline{r})=U_{i}(\underline{r})+\left.\sum_{j} \int_{V} d \underline{r}^{\prime} \frac{\delta^{2}\left(A-A_{0}\right)}{\delta \rho_{i}(\underline{r}) \delta \rho_{j}\left(\underline{r}^{\prime}\right)}\right|_{b u l k} \Delta \rho_{j}\left(\underline{r}^{\prime}\right)
$$

The second derivative term is all that remains to be simplified. Using equation (2.2), the second derivative term becomes

$$
\frac{\delta^{2}(\beta A)}{\delta \rho_{i}(\underline{r}) \delta \rho_{j}\left(\underline{r}^{\prime}\right)}=\frac{\delta \beta \psi_{j}\left(\underline{r}^{\prime}\right)}{\delta \rho_{i}(\underline{r})}
$$

which is the functional inverse of site-site total correlation function 


$$
\frac{\delta \rho_{i}(\underline{r})}{\delta \beta \psi_{j}\left(\underline{r}^{\prime}\right)}=\rho_{i}(\underline{r}) \omega_{i, j}\left(\underline{r}, \underline{r}^{\prime}\right)+\rho_{i}(\underline{r}) \rho_{j}\left(\underline{r^{\prime}}\right) h_{i, j}\left(\underline{r}, \underline{r}^{\prime}\right)
$$

where $\omega_{i, j}\left(r, r^{\prime}\right)$ is the single chain site-site correlation function while $h_{i, j}\left(r, r^{\prime}\right)$ is the correlation function for sites on different chains. Since h(r) is zero for ideal chains, the inverse is easily found to be

$$
\frac{\delta^{2}\left(\beta A_{0}\right)}{\delta \rho_{i}(\underline{r}) \delta \rho_{j}\left(\underline{r}^{\prime}\right)}=\frac{\delta \beta \psi_{j}^{0}\left(\underline{r}^{\prime}\right)}{\delta \rho_{i}(\underline{r})}=\frac{\omega_{i, j}^{-1}\left(\underline{r}, \underline{r}^{\prime}\right)}{\rho_{i}(\underline{r})} .
$$

For the interacting system, the direct correlation function is defined to be the deviation from ideal behavior:

$$
\frac{\delta^{2}(\beta A)}{\delta \rho_{i}(\underline{r}) \delta \rho_{j}\left(\underline{r}^{\prime}\right)}=\frac{\delta \beta \psi_{j}\left(\underline{r}^{\prime}\right)}{\delta \rho_{i}(\underline{r})}=\frac{\omega_{i, j}^{-1}\left(\underline{r}, \underline{r}^{\prime}\right)}{\rho_{i}(\underline{r})}-c_{i, j}\left(\underline{r}, \underline{r}^{\prime}\right)
$$

which when operated upon by its inverse (equation (2.18)) results in the generalized Ornstein-Zernike equation.

The expression for the external field becomes:

$$
\beta U_{i}^{0}(\underline{r})=\beta U_{i}(\underline{r})-\sum_{j} \int_{V} d \underline{r}^{\prime} c_{i j}\left(\underline{r}-\underline{r}^{\prime}\right) \Delta \rho_{j}\left(\underline{r}^{\prime}\right)
$$

This is the hypernetted chain (HNC) form of the ideal external field. Alternate forms of the medium induced potential were also considered. The most successful of these was the Martynov-Sarkisov (MS) field. In the current report, we have restricted our attention to the MS field type.

The surface tension, $\gamma$, can be related to the structure, $\rho(r)$, through the Maxwell relation:

$$
\left(\frac{\partial \gamma}{\partial V}\right)_{T, N, A}=-\left(\frac{\partial P}{\partial A}\right)_{T, V, N}
$$


where $A$ is the surface area. This leads directly to

$$
\beta \gamma=-\int_{0}^{\rho_{\text {Bulk }}} \frac{\Gamma(\rho)}{\rho}\left(\frac{\partial \beta P}{\partial \rho}\right)_{T} \mathrm{~d} \rho
$$

where $\Gamma(\rho)$ is $\int_{0}^{z_{\text {Max }}}\left(\rho(z)-\rho_{\text {Bulk }}\right) d z$; the Gibbs dividing surface is chosen to be at closest approach; and $\mathrm{z}_{\mathrm{Max}}$ is sufficiently far from the wall to be in the bulk region.

\section{Results and Discussion}

We have investigated many different aspects of the DFT theory as applied to Hard Site chains near a hard wall including different field types; different forms for $\mathrm{c}(\mathrm{r})$; and different chain lengths. In the current report we focus on only a few instances.

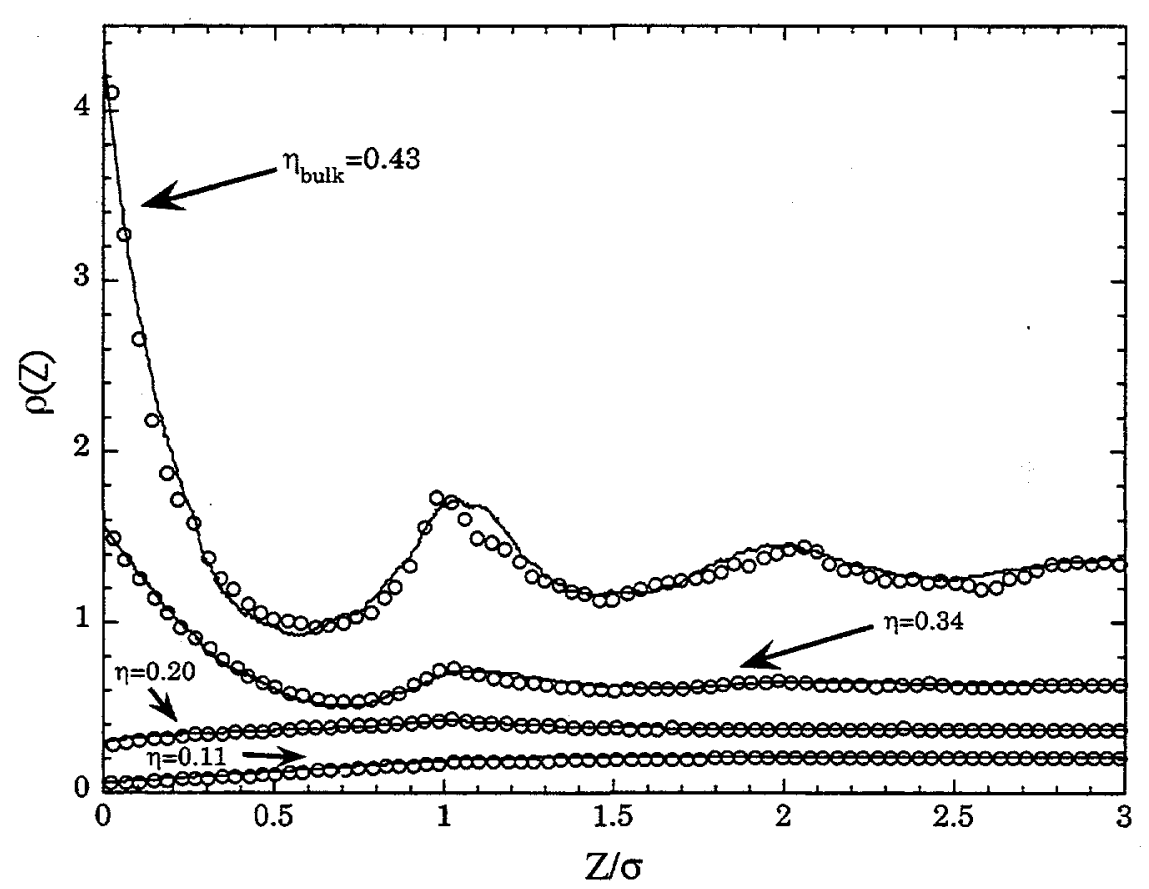


Figure 8. Comparison of Monte Carlo simulations (symbols) with our DFT results. For clarity, the $\eta=0.43$ curve has been shifted up by 0.5 . $\eta=\pi \rho / 6$.

Since our calculation of the surface tension is based upon the density profile, it is important that $\rho(z)$ be accurately calculated by the DF theory. This is shown in Figure 8 where the inhomogeneous density found through DFT theory (for 20 site chains) is compared with those found through full simulations. The effect of the polymeric nature of the molecules on the density profile can be seen by comparing the density profiles of chains ( 20 mers) and monatomics ( 1 mers). This is done in Figure 9 and it is clear that the polymeric nature of the chains reduce the structure induced by the wall. In addition, it is interesting that for this chain model, the period of the density variation is the same as for hard spheres. at a packing fraction, $\eta$, of 0.43 . 


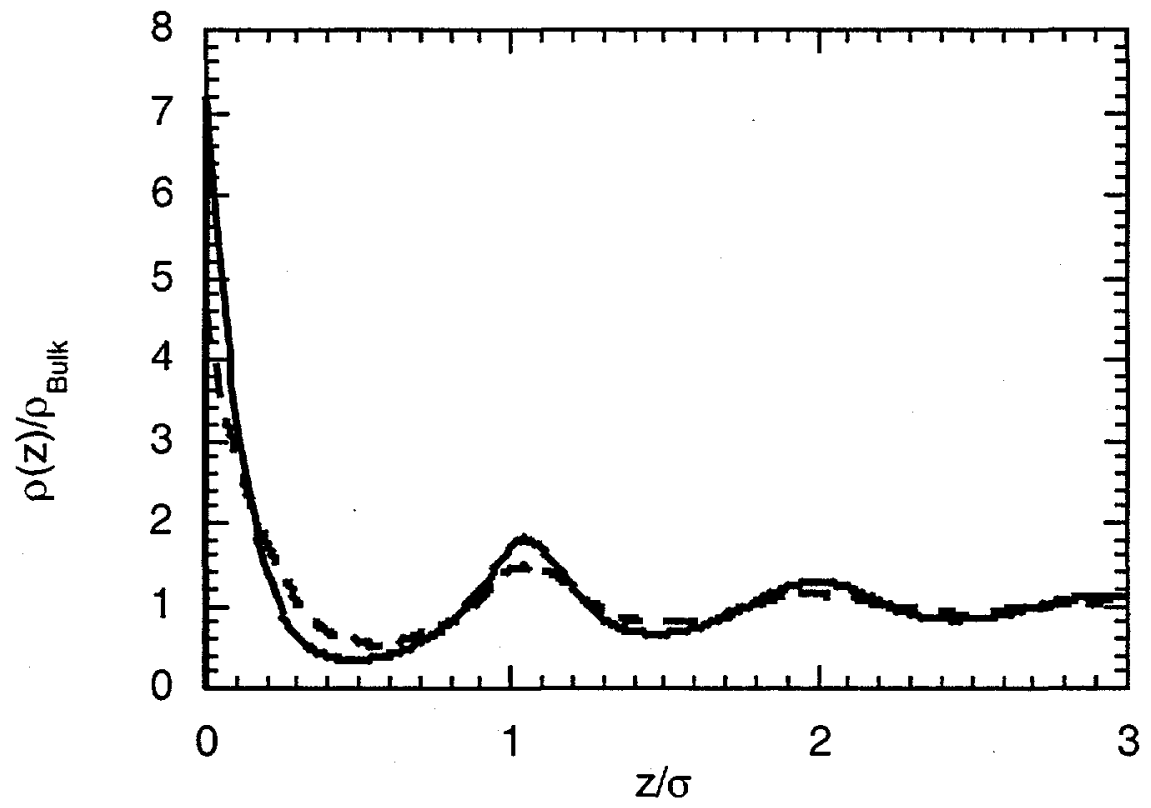

Figure 9 - A comparison between density profiles for monomers (solid line) an $\mathrm{N}=20$ chains (dashed line) obtained from DFT theory.

For hard spheres, the surface excess increases with density for all densities as seen in Figure 10. On the other hand, for chains, the reduction in the single chain entropy near the wall results in a decrease in the surface excess at small densities. At higher densities, the packing entropy dominates, resulting in an increase in the surface excess similar to that seen for hard spheres. Our results are compared with the simulation results shown in Figure 8. The agreement is excellent except at the highest density point where the $z_{\mathrm{Max}}$ of 3 used for the analysis of the simulation results does not capture all the structure. 


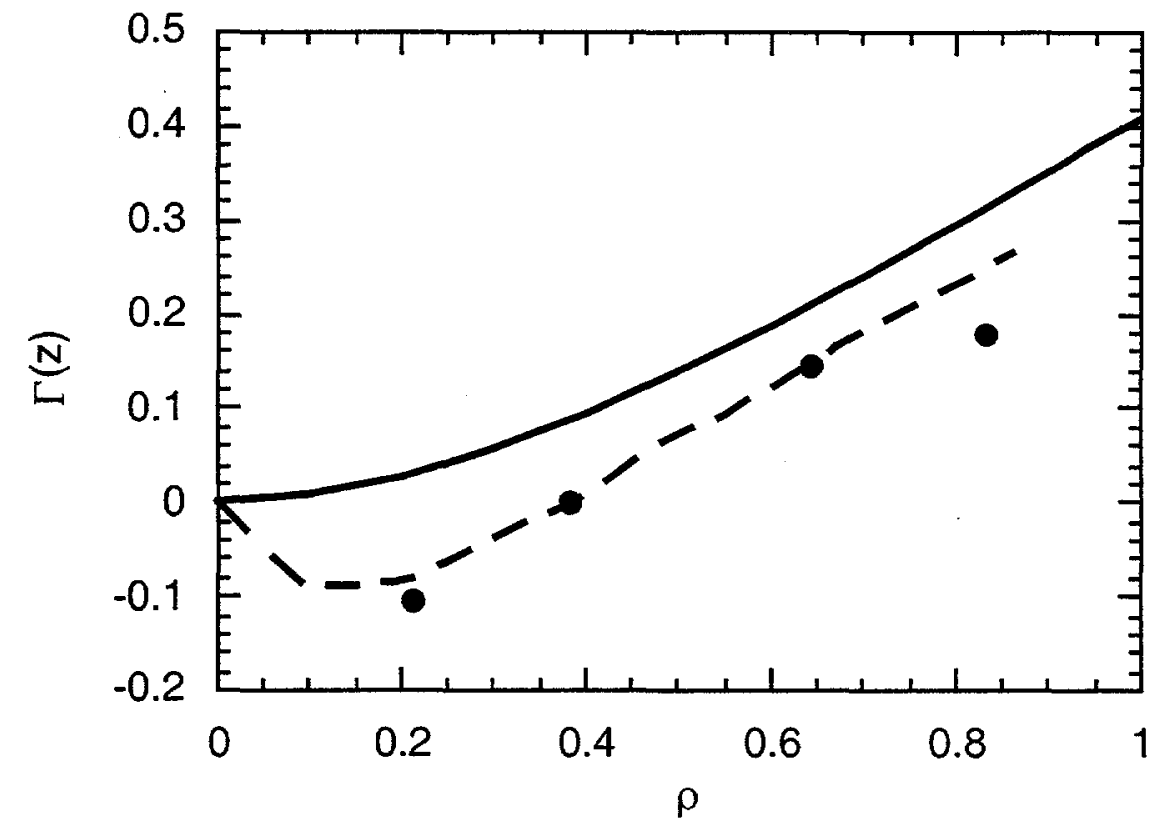

Figure 10. The DF theory predictions of the surface excess of hard spheres (solid line) and of hard chains (dashed line). The symbols are the results of the full simulations of figure 1 (Note that the $\rho=0.83$ point was not integrated to bulk behavior).

In Figure 11, the surface tensions which were calculated from equation (2.23) are compared for hard spheres a 20-mer chains. For the hard spheres, the surface tension steadily decreases for with increasing density. Since the wall decreases the free energy, the atomic, hard sphere liquid will tend to wet the surface. On the other hand, the polymer has a positive surface tension at low densities. Consequently, for a solution of polymers and hard spheres, the monomers will segregate to the wall, and, as a result, the overall adhesion with the wall will be reduced. 


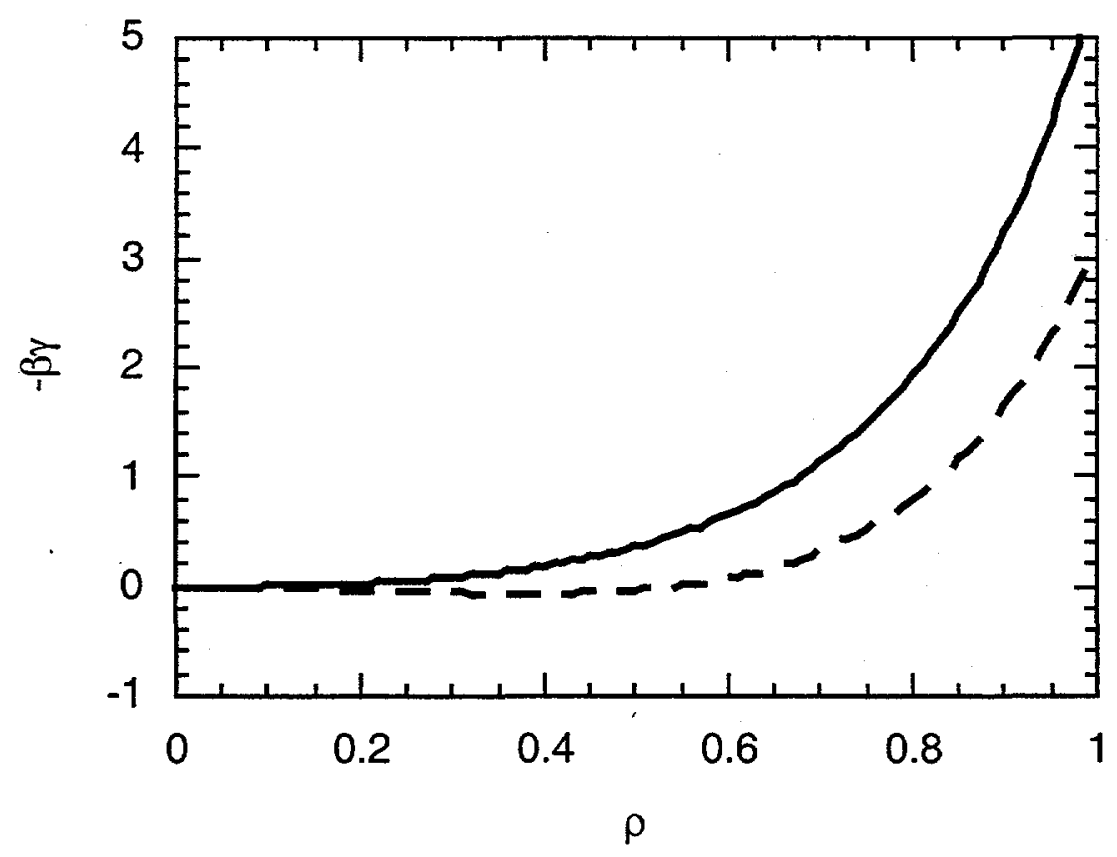

Figure 11. The surface tension for hard spheres (solid line) and 20 mers (dashed line).

We can observe in Figure 11 that the surface tension for the monomer fluid and polymer solution at high concentration is negative. This apparently unphysical result can be explained by realizing that $\gamma$ is not the surface free energy for the air-liquid interface but for the liquid-solid interface. Thus a negative value indicates that the free energy is lowered when the surface is introduced into the bulk liquid. In other words, the liquid tends to wet the surface when $\gamma<0$. Note that in the polymer case that the surface free energy becomes positive at low concentration and then changes sign at higher concentrations. This uniquely polymer effect can be interpreted as a tendency of the solution to de-wet the surface at low concentration of polymer. 
It is tempting to associate this de-wetting tendency with the decreased friction or slipperyness of the solution. We further speculate that when the aqueous solution dewets from the surface, the hydrodynamics of the solution between substrates changes to one where the liquid film "rolls" within the gap. This is in analogy with the well known hydroplaning phenomenon of automobile tires on a wet pavement. This suggests that future work on this problem should focus on experimental measurement of the surface free energy of the solid-polymer solution interface. 


\section{References}

1) J. Hooper, J. D. McCoy, and J. G. Curro,

"Density Functional theory of simple polymers in a slit pore:

1. Theory and efficient algorithm". J. Chem. Phys. (Submitted).

2) J. Hooper, M.T. Pileggi, J. D. McCoy, J. G. Curro, and J. D. Weinhold, "Density

Functional theory of simple polymers in a slit pore:

2. The role of compressibility and field type." J. Chem. Phys. (Submitted).

3) J. Hooper, J. D. McCoy, J. G. Curro, and F. van Swol,

"Density Functional theory of simple polymers in a slit pore:

3. Surface tension." J. Chem. Phys. (to be submitted). 


\section{SECTION 4}

Summary 
This investigation into the "Designed Synthesis of Controlled Degradative Materials" was a very successful research effort. In the area of new materials, one very promising class of super-slick polymers, cross-linked polyacrylamides, was discovered that could used as an alternative to polyethylene oxide materials. In addition, a whole new class of polymers with thermally labile weak-links were developed. These materials change from resins at room temperature to oils at temperatures above $90{ }^{\circ} \mathrm{C}$. The monomers used to prepare these polymers have significant promise in their own right as coupling agents for silica-rubber systems. In the area of mechanical testing, we developed a semi-quantitative test for evaluating super-slick polymers and we investigated controlled degradation of polyethylene oxide through the action of ascorbic acid using viscosity studies. These studies revealed that while ascorbic acid can degrade super-slick properties, the rate of decrease of polymer molecular weight and by inference, lubricity, takes hours even in solution. Finally, computer modeling of the super-slick phenomenon using DFT has provided significant insight into the origin of lubricity to where experiments can now be designed to test the models and eventually lead to further improvements in the materials themselves. 


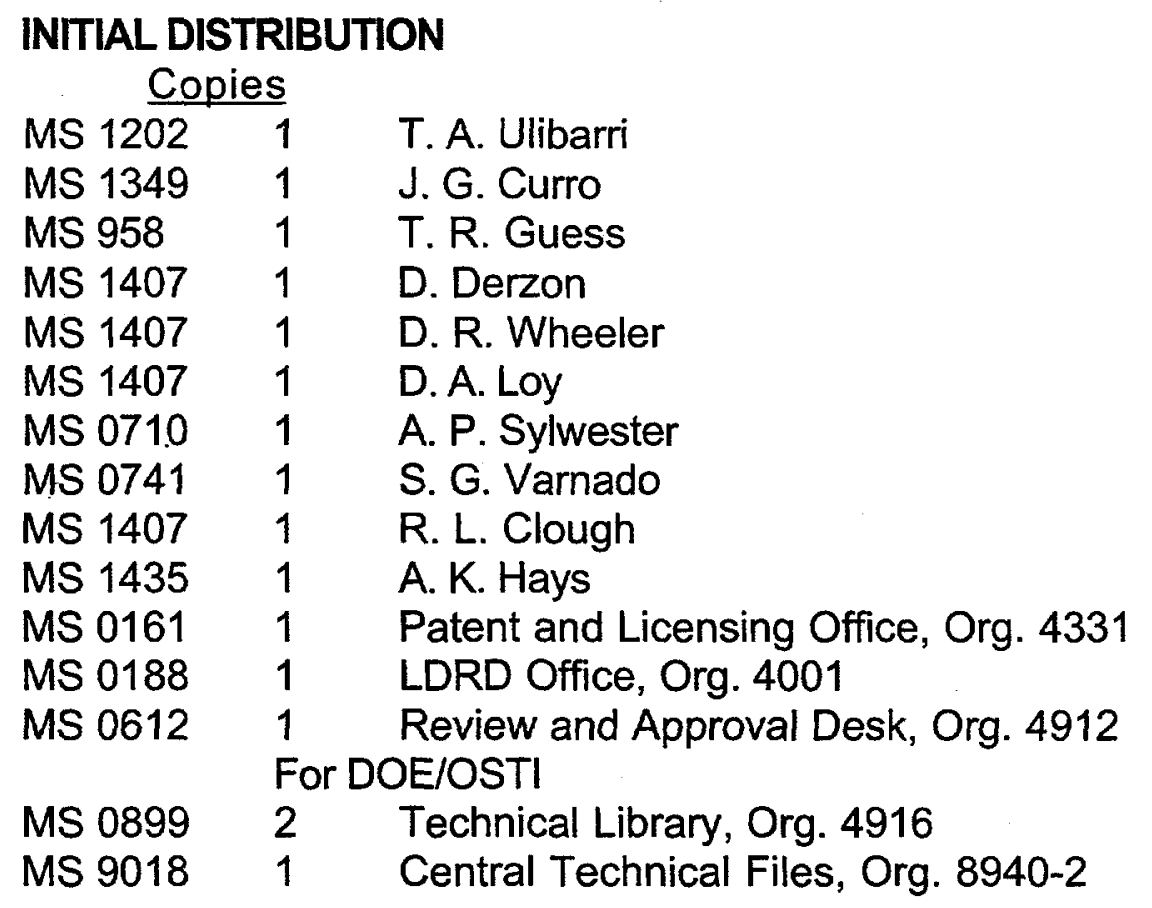

